\title{
Infrared camera as a system visualisation of tumors during resection or biopsy of human's brain
}

by M. Kastek ${ }^{1}$, T. Piątkowski ${ }^{1}$, H. Polakowski ${ }^{1}$, K. Kaczmarska ${ }^{2,3}$,

Z. Czernicki ${ }^{2}$ J. Bogucki ${ }^{2}$, M. Zębala ${ }^{4}$

\author{
${ }_{1}^{1}$ Military Univ. of Technology, Institute of Optoelectronics, Warsaw, Poland, mariusz kastek@wat.edu.pl \\ ${ }^{2}$ Medical Research Centre, Polish Academy of Sciences, Experimental and Clinical Neurosurgery, Warsaw, \\ Poland \\ ${ }^{3}$ Institute of Electronic Systems, Warsaw University of Technology, Warsaw, Poland \\ ${ }^{4}$ Medical Univ. of Warsaw, Department of Neurosurgery, Medical Clinic II, Warsaw, Poland
}

\begin{abstract}
A study of temperature changes across cerebral cortex was performed for five patients with brain tumors (previously diagnosed using magnetic resonance or computed tomography) during surgical resection or biopsy of tumors. Measurements have shown that the temperature on the surface of the cyst was on average $2.6 \mathrm{~K}$ below the temperature of surrounding areas. It has been also observed that during devascularization of a tumor, i.e. cutting off its blood vessels, the tumor temperature lowers significantly in spite of using bipolar coagulation, which causes additional heat emission in the tissue. The results of the measurements are presented on the paper.
\end{abstract}

\section{Introduction}

Intra-cranial tumors are one of most common pathological changes in central nervous system, caused by uncontrolled cell growth in virtually any tissue type. Most common primary tumors (starting in the brain itself) are gliomas, meningiomas, pituitary adenomas and nerve sheath tumors. Apart from primary tumors there can be also metastatic tumors, being the result of cancer spreading from another area of the body. Primary method for the treatment of brain tumors is surgical intervention. Full, complete resection of a benign tumor usually means curing the patient completely, whereas in case of malignant tumors it is only the starting healing procedure, followed by additional treatment in the form of radio- or chemotherapy. Partial tumor resection can also be beneficial to the patient, as it eases the clinical symptoms of the disease [1].

Among many image-based diagnostic methods the computed tomography and magnetic resonance imaging are most popular ones in neurosurgery [2]. Those methods are capable of precise imaging of anatomical structure of the brain, but they are less useful in locating the relevant structures after their displacement due to surgical skull opening and letting off some cerebrospinal fluid. Diagnostic images taken before surgery lose their accuracy and become then quite unreliable. Thermal imaging becomes lately more and more popular diagnostic method in medicine [3, 4].

Operation of a thermal camera relies on the detection of electromagnetic radiation, the source of which is every object at temperature higher than absolute zero $(0 \mathrm{~K})$. Intensity of this radiation is directly proportional to the temperature of the source object [5]. Thermography makes it possible to visualize the temperature distribution across the surface of an observed object. Direct temperature value or temperature difference can be treated as useful information signal. In medical applications the tissue areas with higher metabolism and blood supply are indicated as hotter ones.

The goal of the undertaken research is to assess the capability of a thermal camera to identify the borders of brain tumors and to develop methods of intraoperative image-based monitoring for the analysis of temperature changes in the cerebral cortex.

\section{Clinical tests}

Temperature changes on the surface of cerebral cortex were analyzed in 6 cases (5 males and 1 female patients), all having previously diagnosed cancer (by computed tomography and magnetic resonance methods) and initially qualified for biopsy or tumor resection. Mean age of a patient was 61 years (between $38-82$ years of age). Analyzed brain cancer cases can be classified, by their etiology, into gliomas, meningiomas and metastatic tumors.

Measurement were conducted using SC5600 thermal camera (FLIR, USA) with $54 \mathrm{~mm}$ lens (field of view $10^{\circ} \mathrm{x}$ $8^{\circ}$ ). This camera is fitted with cooled InSb detector array with temperature resolution of $25 \mathrm{mK}$ and operates in MWIR band of infrared spectrum (wavelength from $3 \mu \mathrm{m}$ to $5 \mu \mathrm{m}$ ). All of the measurements were conducted in Neurosurgery Clinic of Warsaw Medical University. The clinical tests started after the consent had been obtained from Bioethics Commission of Warsaw Medical University (document No. Kb/178/2013).

Thermal camera was properly sterilized and placed approximately 2 meters from operating table, thus it had no direct contact with operating environment or medical crew. Neurosurgical procedures were carried out in general anesthesia with craniotomy access to the brain surface. Finally the surface of cerebral cortex has been revealed after 


\subsection{1/qirt.2016.055}

cutting through the pia mater. The temperature distribution across the observed brain area was monitored continuously in real-time regime.

\section{Measurement evaluation}

All pathological changes differ in metabolism form healthy tissues, thus the temperature difference occurs, which can be used to identify the affected area. The paper present preliminary results obtained during thermal imaging of intracranial metastatic tumor. Fig. 1 shows initial recording of temperature distribution on the surface of the pia mater, after cutting through dura mater and arachnoids mater. At each stage of neurosurgical procedure the relative temperature distributions were recorded, not the absolute ones. It was not possible to determine the absolute temperature values because the emissivity of visualized area of cerebral cortex were constantly changed by saline flow applied to it, which significantly disturbed the measurements.
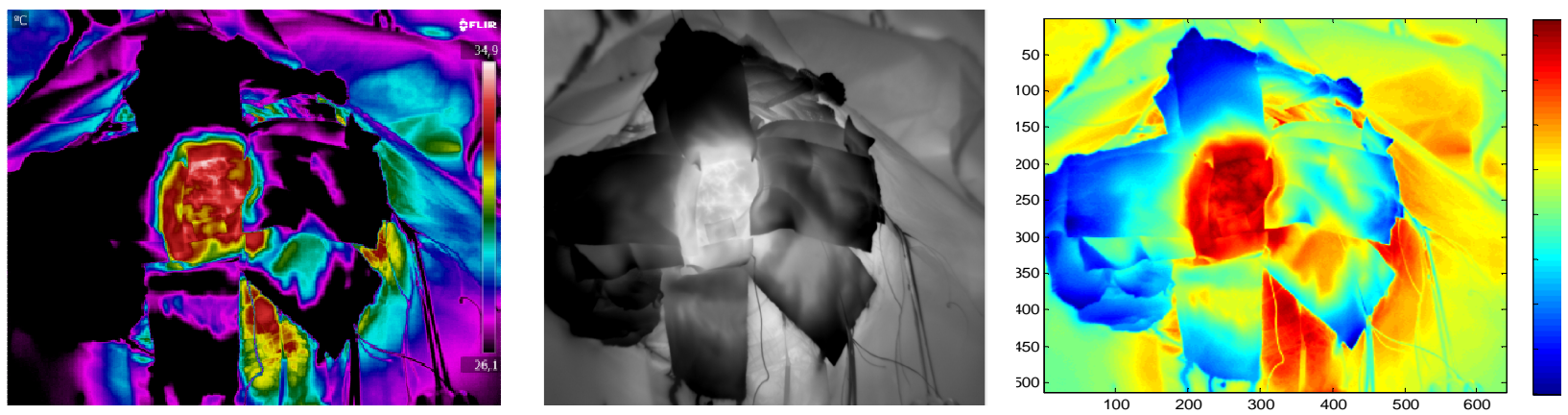

Fig. 1. Recorded thermal images of operating area, with pia mater intact (prior to tumor resection).

In the case of metastatic tumor with large edema and a liquid-filled space different temperature of a cerebral cortex were recorded depending on metabolic activity. Measurements have shown that the temperature on the surface of the cyst (measurement points Sp1 and Sp5 in Fig. 2c) was on average 2.6K below the temperature of surrounding areas.

(a)

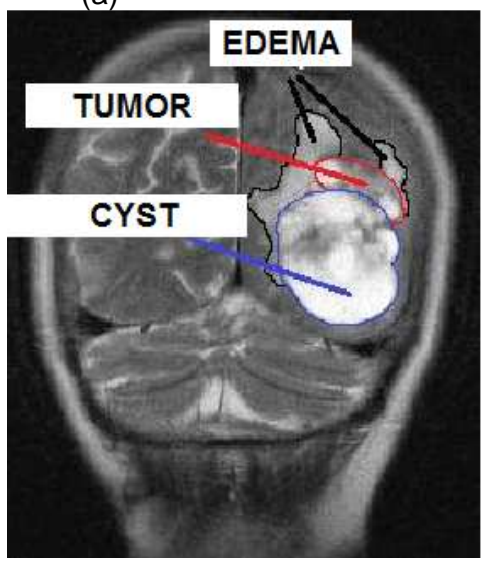

(b)

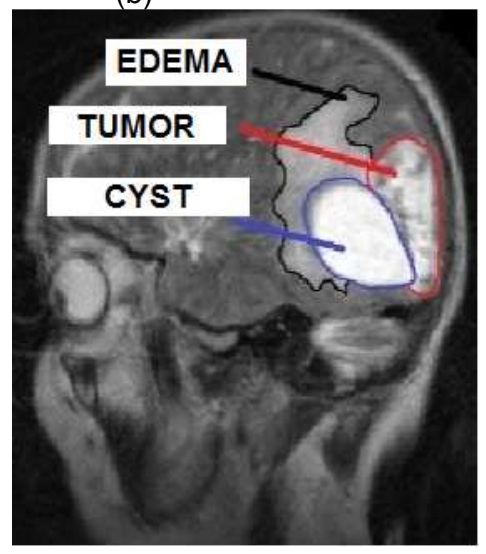

(c)

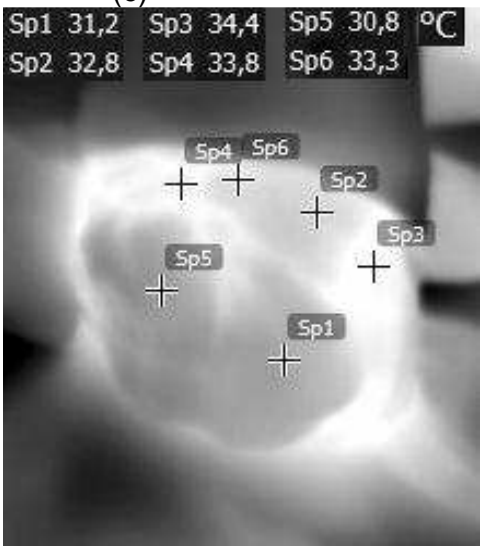

Fig. 2 Magnetic resonance (coronal plane, T2 view) (a), magnetic resonance (sagittal plane, T2 view) (b) of a patient with metastatic tumor with large cystic space; thermogram of the operating field registered before tumor resection with marked measuring points (Sp1 and Sp5 - cyst surface, Sp2, Sp3 - area of solid tumor, Sp4, Sp6 - edema surface) (c)

\subsection{Thermal data analysis for metastatic (lung cancer) tumor}

In case of the metastatic tumor with a large edema and a cyst (fluid-filled space), different temperatures were recorded on the surface of a cerebral cortex, depending on metabolic activity. The results are shown in Fig.3. 


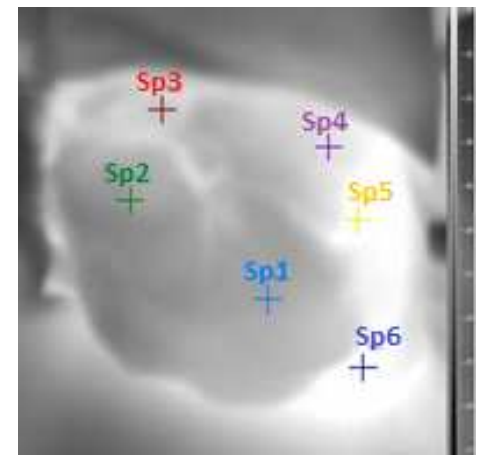

a)

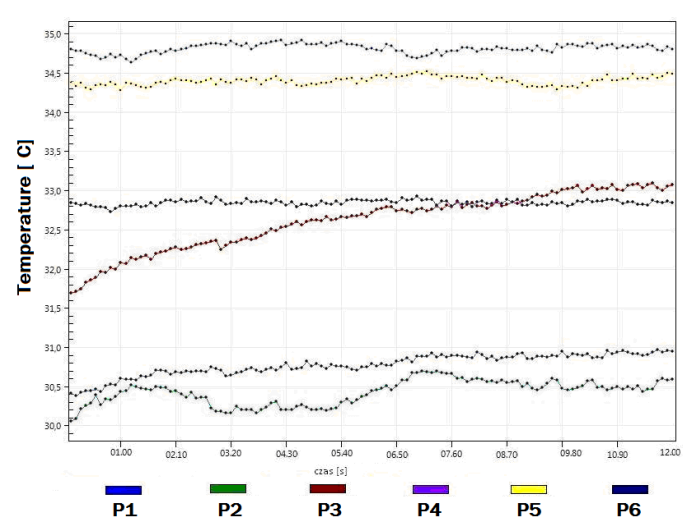

b)

Fig. 3. Thermal image of operating area with marked measurement points (a) and corresponding temperature changes over time before tumor resection (b).

Analysis of the recorded thermal images revealed, that the cyst surface temperature (points 1 and 2, Fig. 3) was on average $2^{\circ} \mathrm{C}$ lower than surrounding area (probably edema area) and $4^{\circ} \mathrm{C}$ lower than the temperature of cancerous tissue. Similar measurements and analysis of temperature changes on the surface of cerebral cortex was performed (in selected points) during tumor resection.

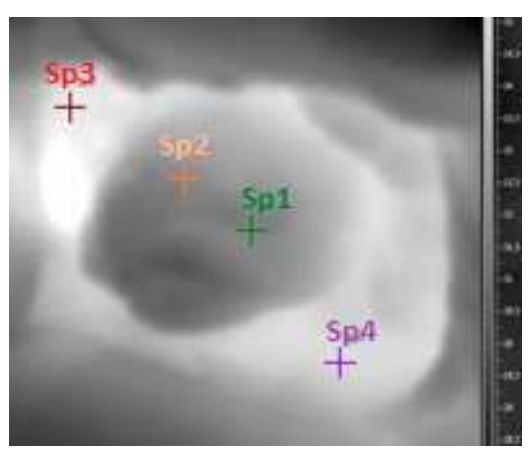

a)

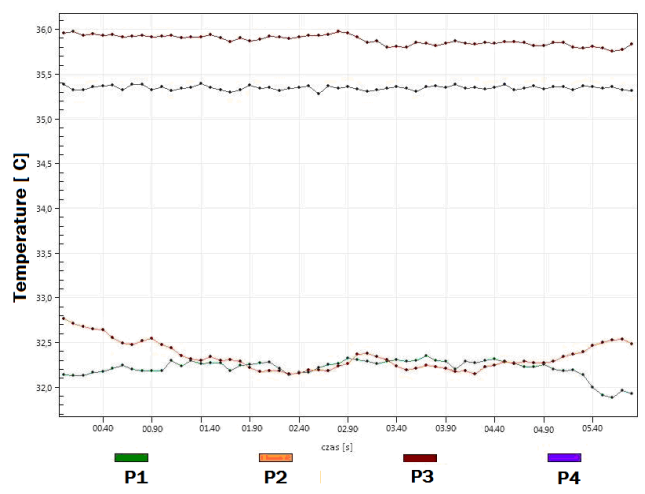

b)

Fig. 4. Thermal image of operating area with marked measurement points (a) and corresponding temperature changes over time during tumor resection (b).

Analysis of the recorder temperature data allowed for identification of the borders of cancerous tissue and healthy cerebral cortex. During devascularization (cutting-off blood supply vessels) a significant tumor temperature drop was observed, even though the procedure was performed using bipolar coagulation which itself results in a heat emission inside a tissue (Fig. 4). Average temperature values were also calculated for selected areas and results are presented in Table 2.

Table 2. Temperature (averaged over 2-minutes period) and standard deviation values for selected brain areas measured before tumor resection.

\begin{tabular}{|l|l|l|l|l|}
\hline ROI & $\begin{array}{l}\text { Tmin } \\
{\left[{ }^{\circ} \mathrm{C}\right]}\end{array}$ & $\begin{array}{l}\text { Tmax } \\
{\left[{ }^{\circ} \mathrm{C}\right]}\end{array}$ & $\begin{array}{l}\text { Tmean } \\
{\left[{ }^{\circ} \mathrm{C}\right]}\end{array}$ & $\begin{array}{l}\text { STD } \\
{\left[{ }^{\circ} \mathrm{C}\right]}\end{array}$ \\
\hline 1 & 29.45 & 33.25 & 30.96 & 0.78 \\
\hline 2 & 31.61 & 33.94 & 32.56 & 0.52 \\
\hline 3 & 32.25 & 34.95 & 34.09 & 0.49 \\
\hline
\end{tabular}

\subsection{Thermal data analysis for metastatic (lung cancer) tumor during devascularization procedure}

Thermal image recorded during devascularization procedure performed by bipolar coagulation method is shown in Fig. 5. Application of bipolar coagulation is generally beneficial in case of all medical procedures requiring particularly careful approach, such as neurosurgery, but also in otolaryngology, gynecology and minimally invasive surgery. Coagulation is performed using bipolar forceps with integrated electrodes. Electric current flows only in the limited tissue 


\subsection{1/qirt.2016.055}

volume between the electrodes and not through entire patient's body. As opposed to monopolar electrosurgery, the grounding is not required and the potential risk factors are also significantly reduced. The current leakage is very small, there is minimal risk of electrical burn for the patient and electrical interference level is much smaller, thus the operation of pacemakers or ECG monitors (essential in neurosurgical procedures) is not interrupted.

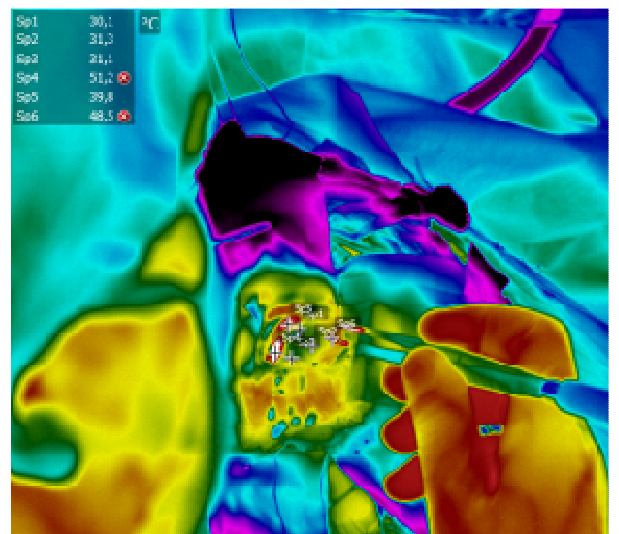

Fig. 5. Devascularization of cancerous tissue viewed by a thermal camera

Fig. 67 illustrates the changes of mean temperature and standard deviation values in the selected region of interest ( $\mathrm{ROI} 1$ - cyst, ROI 2 - edema, ROI 3 -cancerous tissue) just before tumor resection.
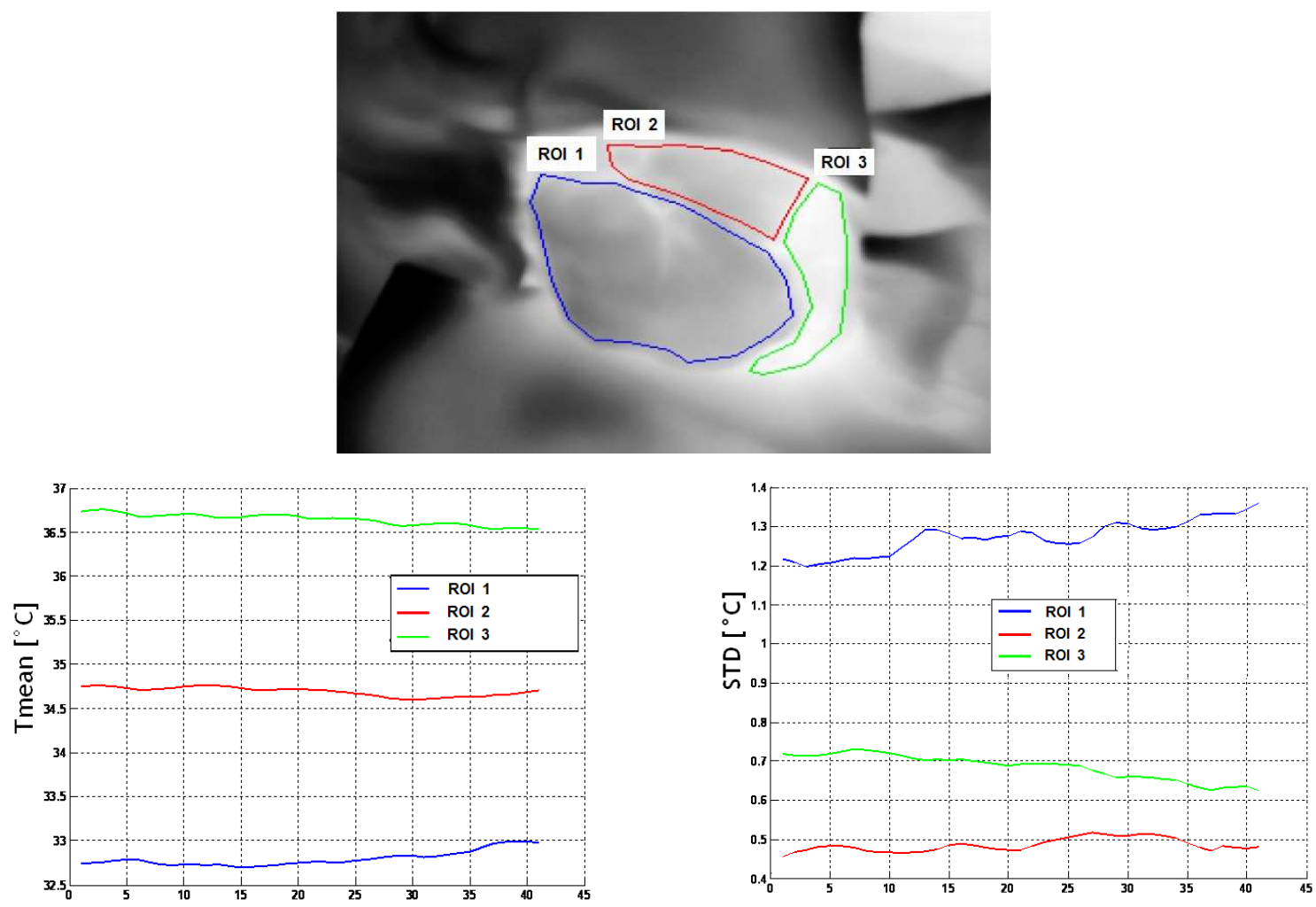

Fig. 6. Temperature changes and calculated standard deviation values in selected areas of cerebral cortex before tumor resection.

Distinct temperature differences were observed between selected areas. Lowest temperature was recorded in cyst area. Peak value of temperature difference between cyst and cancerous tissue reached $5.5{ }^{\circ} \mathrm{C}$. Higher standard deviation of temperature in the cyst region is caused by the presence of blood vessels there. Blood flow causes temporary changes in metabolic activity, which in thermal imaging is manifested as temperature fluctuations. 
Table. 3. Temperature (averaged over 10-minutes period) and standard deviation values for selected brain areas measured during tumor resection.

\begin{tabular}{|l|l|l|l|l|}
\hline ROI & $\begin{array}{l}\text { Tmin } \\
{\left[{ }^{\circ} \mathrm{C}\right]}\end{array}$ & $\begin{array}{l}\text { Tmax } \\
{\left[{ }^{\circ} \mathrm{C}\right]}\end{array}$ & $\begin{array}{l}\text { Tmean } \\
{\left[{ }^{\circ} \mathrm{C}\right]}\end{array}$ & $\begin{array}{l}\text { STD } \\
{\left[{ }^{\circ} \mathrm{C}\right]}\end{array}$ \\
\hline 1 & 30.65 & 31.48 & 31.06 & 0.88 \\
\hline 2 & 34.19 & 34.39 & 34,27 & 0.52 \\
\hline 3 & 32.31 & 32.75 & 32.59 & 0.44 \\
\hline
\end{tabular}

Fig. 7 shows the sample thermal image recorded during tumor devascularization by bipolar coagulation method.
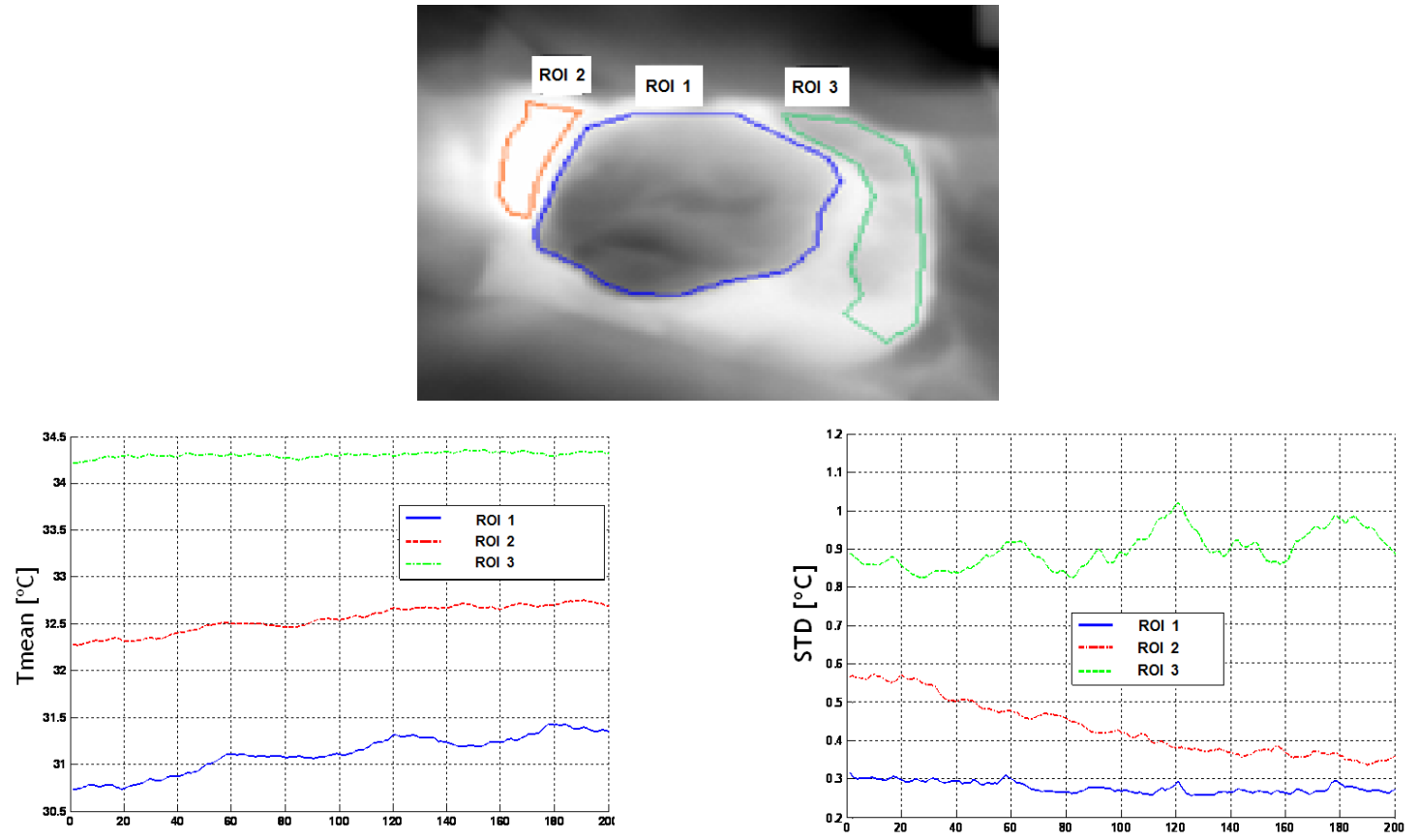

Fig. 7. Temperature changes and calculated standard deviation values in selected areas of cerebral cortex during tumor resection

During the process a significant difference in mean temperature values can be observed between cancerous tissue and surrounding areas. Coagulation process causes local temperature rise in the processed tissue (ROI 1). In areas ROI 2 and ROI 3 (edema and healthy tissues, respectively), the mean temperature value is higher than in tumor (by $1.5^{\circ} \mathrm{C}$ ) and in cyst area (by $3.5^{\circ} \mathrm{C}$ ). This difference is caused mainly by decreased metabolism in the tumor caused by devascularization, as the blood supply to cancerous tissue gets gradually reduced.

\subsection{Image processing method}

An attempt to distinguish and precisely locate the borders of cancerous and healthy tissues was also undertaken, by applying image processing and image enhancement methods to the recorded thermal images. The thermal image containing data on temperature distribution across observed area of cerebral cortex was saved as RGB color palette image. Then, in order to improve contrast, an image conversion was performed using LUT (look-up table) method. Coefficients in the applied LUT were calculated according to the following relation:

$$
\operatorname{LUT}(i)=\left\{\begin{array} { c } 
{ 0 } \\
{ a ( i - \frac { i _ { \operatorname { m a x } } } { 2 } ) + \frac { i _ { \operatorname { m a x } } } { 2 } } \\
{ i _ { \operatorname { m a x } } }
\end{array} \text { if } \left\{\begin{array}{c}
a\left(i-\frac{i_{\max }}{2}\right)+\frac{i_{\max }}{2}<0 \\
0 \leq a\left(i-\frac{i_{\max }}{2}\right)+\frac{i_{\max }}{2}<i_{\max } \\
a\left(i-\frac{i_{\max }}{2}\right)+\frac{i_{\max }}{2}>i_{\max }
\end{array}\right.\right.
$$

where $i_{\max }$ is maximum allowed value for RGB component of image pixel. 
If the value of an a constant (interpreted as the slope of a straight line) is greater than 1 , then contrast increase will occur. In the opposite case (a smaller than 1) contrast will be decreased. The value of a constant was determined by analyzing the histogram of RGB image components. Result of image processing by aforementioned method is shown in Fig. 9a.

In the next step of image processing the Sobel operator was applied for edge detection. It is performed by matrix convolution in order to find the edge direction for every pixel. Each neighborhood pixel carries its own weight value to the calculations. Those weights are written in a form of two $3 \times 3$ kernels, one for horizontal and one for vertical directions. For a Sobel operator those kernels are as follows:

\section{SOBEL MASK}

\section{HORIZONTAL}

$\begin{array}{rrr}1 & 2 & 1 \\ 0 & 0 & 0 \\ -1 & -2 & -1\end{array}$

\section{VERTICAL}

$\begin{array}{lll}1 & 0 & -1 \\ 2 & 0 & -2 \\ 1 & 0 & -1\end{array}$

Fig. 8. Horizontal and vertical kernels of Sobel operator

Figure 9a shows the thermal image after the Sobel operation, whereas Figure 9b presents the result of contrast enhancement and histogram analysis. Both methods allow for determining the boundaries between cancerous and healthy tissues.

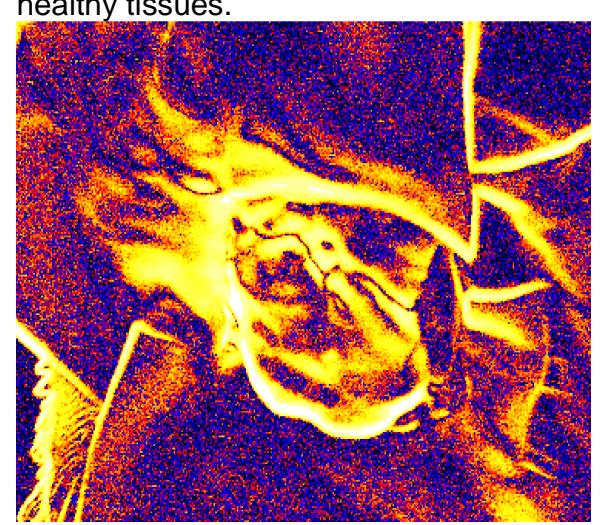

a)

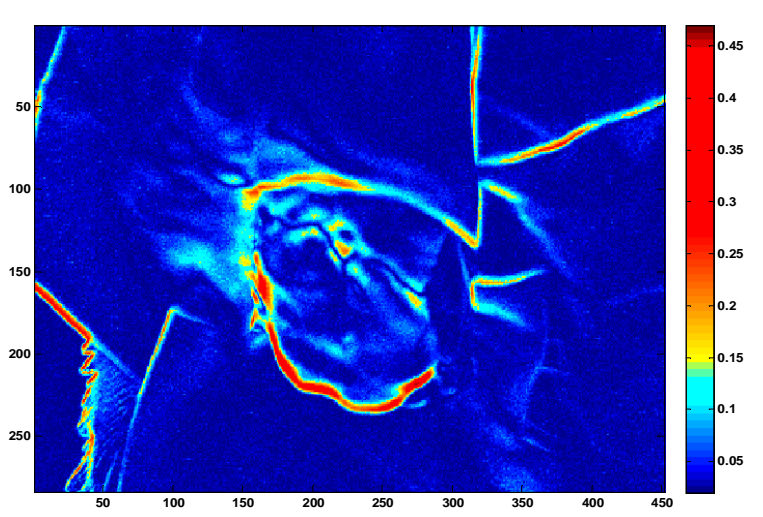

b)

Fig. 9. Results of image processing

\section{Conclusion}

Initial analysis shows that during resection temperature of normal tissue is significantly higher $(p=0.028943)$ than temperature of metastatic tumor. Similar results were obtained for temperature of normal and pathological tissues before resection. Temperature of metastatic tumor during devascularization is $30.80 \pm 1.64{ }^{\circ} \mathrm{C}$ and temperature of normal tissue is $33.49 \pm 1.14{ }^{\circ} \mathrm{C}$ (median $\pm \mathrm{QR}, \alpha=0.05$ ). We can observe that difference between temperature of normal and pathological tissues increases during resection. It is caused mainly by decreased metabolism in the tumor caused by devascularization, as the blood supply to cancerous tissue gets gradually reduced.

Results of intraoperative measurement confirmed the usability non-invasive thermal imaging to record cerebral cortex temperature changes. Thermal camera can be effectively used to assess the blood supply of both healthy and pathological tissues. Thermal images recorded during neurosurgery allow to locate and determine the brain tumour borders, which is extremely important in order to precisely resect tumour and minimize the neurological deficits occurring after the surgical procedure.

\section{REFERENCES}

[1] Greenberg M. S. „Handbook of neurosurgery”, Thieme, 7th Edition, 2010

[2] Walecki J. „Postępy neuroradiologii”, Polska Fundacja Upowszechniania Nauki, Warszawa, 2007

[3] Kateb B., V. Yamamoto, C. Yu, „Infrared imaging: A review of the literature and a case report”, Neurolmage, Vol. 47 (2009)

[4] Ring E. F. J., Ammer K., „Infrared thermal imaging in medicine”, Physiol. Meas., Vol. 33 (2012)

[5] Madura H. „Pomiary termowizyjne w praktyce”, PAK, Warszawa, 2004

[6] Kateb B., V. Yamamoto, C.Yu, W. Grundfest, J. P. Gruen, Infrared thermal imaging: A review of the literature and case report, Neurolmage 47, pp. 154-162, (2009). 